\title{
AN EFFICIENT METHOD FOR CREATING MULTIMEDIA COURSEWARE
}

\author{
Yukinori Terahama ${ }^{\dagger}, \mathrm{Hiroyuki} \mathrm{Kojima}^{\dagger}$ and \\ Hiroh Yamamoto \\ †Systems Development Laboratory, Hitachi \\ Ltd., \\ \#Hitachi Electronics Services Co., Ltd. \\ Kansai Bldg.8-3-45 Nankouhigashi, Suminoe, \\ Osaka, 559 Japan, \\ Tel:+81-6-616-1108 Fax:+81-6-616-1109 \\ E-mail:\{terahama,kojima\}@sdl.hitachi.co.jp
}

\begin{abstract}
In recent years, applications in the field of education have been increasing due to pervasive internet technologies. One particular area of study has been on an efficient method for creating courseware, in order to achieve remote education through networking technology. In this paper, we propose a method for creating courseware by the reuse of previous material. We show that the efficiency of the method is about three fold better than that of the conventional method in creating courseware.
\end{abstract}

Keywords

CAI, Courseware, Efficient method, Network, Multimedia 


\section{Introduction}

Multimedia learning is attractive for use in CAI division support systems which make the most of several media including reflection, shape, sound, and text. Multimedia PCs offer several educational functions in only one PC machine [Fukuhara 91] [Fukuhara 93]. These systems employ frequently internet technologies that have appeared in recent years. In particular, they provide a learning support environment using the WWW(World Wide Web), and are designed to be applied to ITS and authoring environments employing hyper-link [Maruyama 95].

The subject of efficient courseware editing is highlighted to enable various courseware to be offered quickly, in order to achieve remote education at many locations utilizing networking technology. More specifically, in the educational division with one by one renew contents for dizzy progress of technology likely maintenance of PC it takes time to create courseware and to attain the educational objective. For efficient development of courseware, we propose a method that reuses past courseware data to create new high-quality courseware. We compare the new courseware creating method with a conventional one. An experiment was done to verify the educational effect of the proposed method in CAI education. Experiment results show that the efficiency in courseware development is improved about three times compared with the conventional method.

\section{Courseware Using A Method Involing Templates}

Templates have a structure to allow the reuse of past courseware and material data. In creating new courseware, new courseware structures are made by reusing template courseware. From the reuse of template material data, a display frame is made to improve the making of the courseware.

\subsection{Template refinement of courseware structures}

We created a template frame for a courseware structure that included "Chapter", "Paragraph" and "Term" levels, by reusing already made courseware. A structured courseware template was used to make the new courseware frame. The reused sample of the structured courseware is shown in Figure 1.

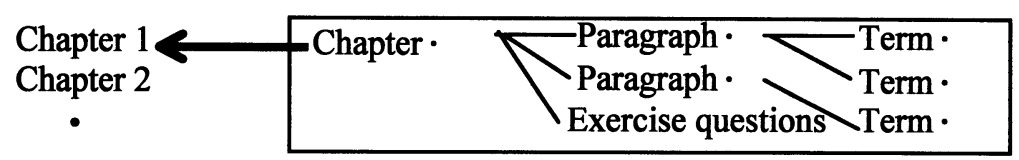

Fig. 1: Reuse of template courseware

Comprehension is simplified if a new courseware structure is made. In the above example, the template can design courseware to satisfy "Chapter", "Paragraph", and "Term", and a related display "Frame". 


\subsection{Template refinement of courseware material data}

A "Frame" applicable to the display of courseware with small changes can be used for efficient design. To achieve this, material data (i.e., existing text and image (movie, picture) data), we search for the courseware necessary for this task in a database, and then we design a new "frame" by redesigning material data.

\subsection{Courseware creating function}

Figure 2 shows several functional structures to create courseware (like that described in chapter 3.2), and we describe the details of several functions below.

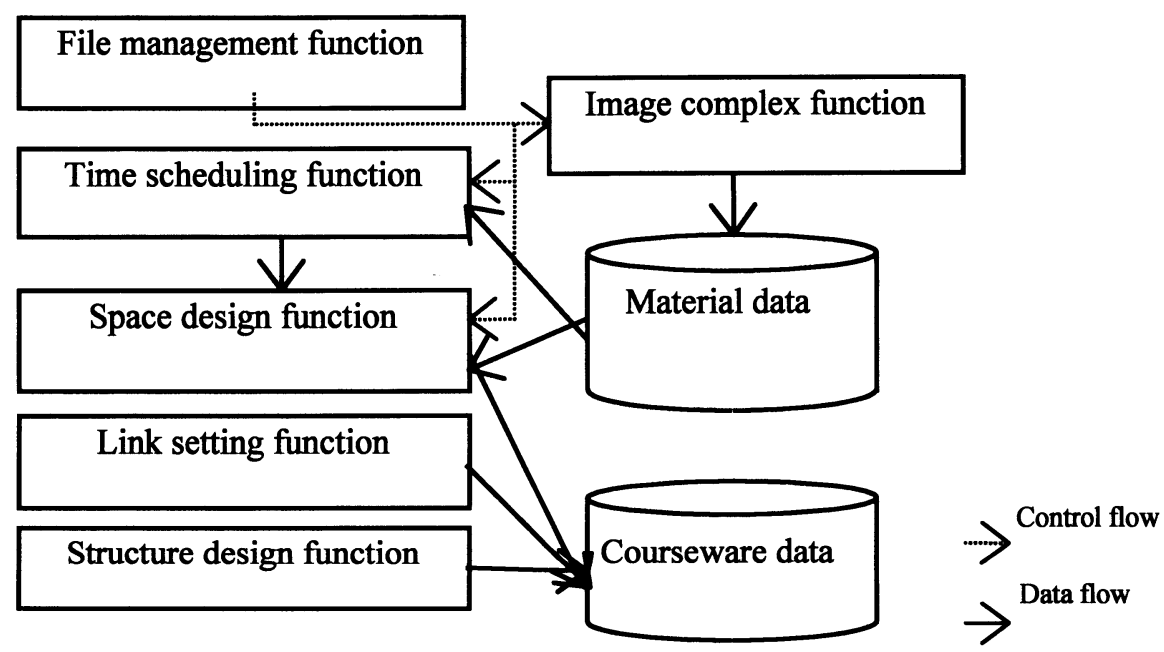

Fig. 2: Structure of courseware creating function 
2.3.1 Structure design function This function is for designing the courseware structure in order to satisfy "Chapter", "Paragraph", and "Term". Designing the new courseware structure is handled as the refinement of the parts template the courseware structure. This function involves partial copying, moving and deleting.

2.3.2 Image complex function This function is for creating a new material data by the template refinement of the material data involving the image data. The newly created material data can be reused by performing template refinement again.

2.3.3 Space design function This function is for creating a display location for the material data, i.e., the image data, etc., made by the image complex function on the "frame". With this function, some part of the plural image data set into the same "Frame" can be registered in the courseware database for added efficiency in the making of the ."Frame", and can be reused by being copied or move to another "Frame". The difference between this function and the image complex function is this point of being copied or moved to another "Frame".

2.3.4 Time scheduling function This function is for scheduling the "Frame" display time, i.e., how long to display the time chart for creating the "Frame". In the scheduling of the display time, this function sets the display start time of several "Frames", and sets the display time for how long the subsequent time will be. It also sets the synchronisation between animation frames, where format and sound data are sent.

2.3.5 File management function This function is for managing image data files called from the image complex function, the space design function, and the time scheduling function. With this function,it is easy to edit efficiency edit courseware by copying or moving file units.

2.3.6 Link setting function This function is for setting links between "Frames". In setting links, it displays a "Frame" list of link connections, and sets links efficiency by verifying the contents of the "Frame" displays.

\section{Evaluation Of The Courseware Creating Efficiency}

We created courseware for an ATM (Automatic Teller Machine) and presumed how long the same task would take in terms of man-hours using the conventional CAI creating method. The proposed method improved the efficiency about three times in comparison with the conventional method.

\subsection{Conclusion on the man-hours for creating the ATM courseware}

We compared the CAI courseware creating procedure for PC maintenance and that for an ATM, to evaluate the courseware creating man-hours for the ATM. We created courseware under a common scenario, and made the ATM courseware with 30 frames. 
Table 1: Conclusion on the man-hours for creating

\begin{tabular}{|l|l|c|c|}
\hline \multicolumn{2}{|c|}{ Objective courseware } & CAI for PC (140 frames) & CAI for ATM (30 frames) \\
\hline Steps & 5-man-months & - \\
\hline Text creation & 22 -man-months & 8 hours (22 hours) \\
\hline $\begin{array}{l}\text { All-around } \\
\text { design }\end{array}$ & Scenario creation & 44-man-months & 15 hours (44 hours) \\
\hline $\begin{array}{l}\text { Detail } \\
\text { design }\end{array}$ & Making of frames & 9-man-days & 1 hour (10.5 hours) \\
\hline $\begin{array}{l}\text { Data } \\
\text { Creating }\end{array}$ & Video data creation & 10-man-days & 9 hours \\
\cline { 2 - 4 } & Graphic data making & 10-man-days & 12.5 hours \\
\cline { 2 - 4 } & Text data making & 15-man-days & 22.5 hours \\
\hline
\end{tabular}

Several working steps were used in creating the text, i.e., all-around design, detailed design, data creating, and test (verification). In Table 1, for the PC courseware involving the typical CAI creating method for text creation, we spent 5-manmonths to make the courseware, ATM courseware with the typical CAI creating method because a scenario has already been made. In creating the scenario for the all-around design and creating frame seats in the detailed design, for the CAI making method, we spent 22-man-days and 44-man-days. On the other hand, for the ATM courseware, the all-around design involved 8 hours and the detailed design involved 15 hours. However, the former 8 hours were needed to set an address for analysing already made courseware. Moreover, the letter 15 hours the appropriate new courseware to make, in place of the existing video data.

Therefore, we assumed the presumed value of the creation time, for the allaround design and detailed design of the ATM courseware, to be 22 hours and 44 hours. (a value in brackets in table 1 is a presumed value).

\subsection{Comparison of ATM courseware creation man-hours}

Table 2 shows results comparing the conventional creating method and the CAI courseware creating method (i.e., the proposed method).

In Table 2, we found that this proposed method shortens the time about threefold compared with the CAI creating method. We analysed the comparison on the working man-hours in several steps as shown in Table 2.

3.2.1 All-around design After we did the all-around design from scratch, we compared with the working man-hours of the CAI courseware using typical image data, about 22 hours were spent. On the other hand, the courseware creation manhours by the proposed method were about $1 / 3$ the man-hours. We presumed the main factor as the creation efficiency with the improved design method in a topdown manner on the assumption that the courseware was structured by "Chapter", "Paragraph", and "Term". 


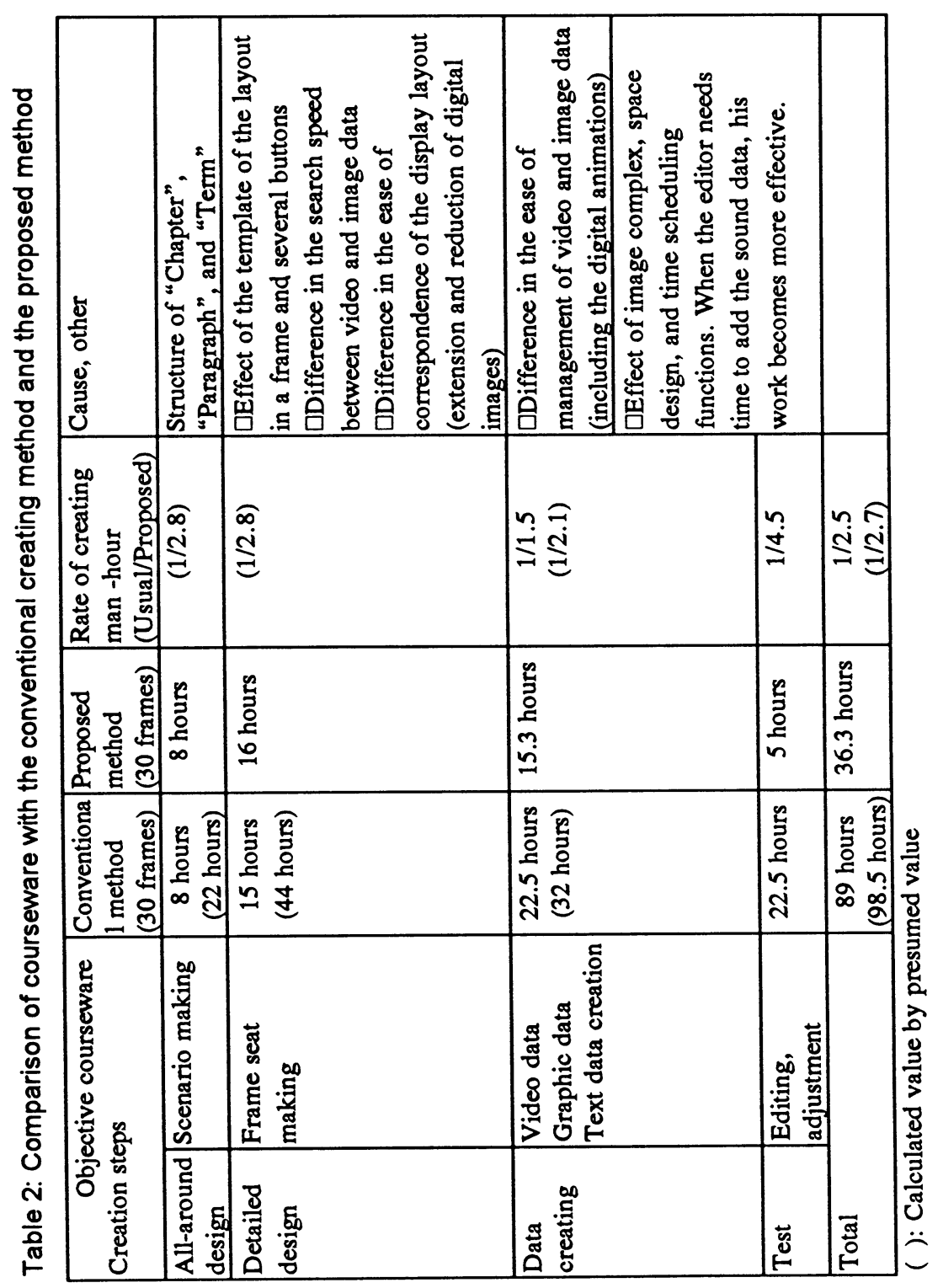


3.2.2 Detailed design Similar to the all-around design, the courseware creation man-hours by the proposed method were about $1 / 3$ the man-hours of the conventional method. We presumed the main factor as the creation efficiency with the improved searching speed from the image database (image data), magnification and reduction were made easier by using the image complex function of this proposed method.

\subsubsection{Data creating}

3.2.3.1 Creating video data In the CAI courseware assuming typical image data, when we created video, the courseware creation efficiency improved by the proposed method. We presumed the main factor as the improved creation efficiency, the movie was structured by multiple images like animation sending frames and data editing was easy.

3.2.4 Creating graphic, data creating The man-hours for the CAI courseware using typical image data, i.e., in creating the graphic data, almost agreed with the creation man-hours of the proposed method. We presumed the main factor as the agreement of the CAI system using the typical image data generated from the use of a depiction tool from an application program, almost the same man-hours as for the image complex function were needed with the proposed method.

3.2.5 Creating text data Because the man-hours for creating the CAI courseware using typical image data, i.e., in creating the text data, were necessary to superimpose the text data on the image display by LD, was taken time to rectify the display timing of the text data. On the other hand, in creating the text data with the proposed method, because the image complex function could be used to edit one frame in which the image data and text data were together, it was not necessary to worry about the above superimposing or display timing and because of this, the courseware creating function was separated to improve the efficiency.

3.2.6 Test In the test (edit and modification step) we presumed that the creation efficiency of the courseware improved because the proposed method was separated by the image complex function, the space design function, and the time scheduling function (like in creating the text data). Moreover, with this proposed method if we needed improved timing to add sound data to the courseware, the creation efficiency improved by the time scheduling function.

Consequently we compared the man-hours for creating the courseware in Table 2 and the most efficient creation step to create the text data. Moreover, it became clear that this contributed toward improving the efficiency of the editing and modification step in the test. In all cases of, we presumed the effect of the proposed method by separating the image complex function, the space design function, and the time scheduling function.

\section{Conclusion}

- By the proposed courseware creating method, the courseware creation efficiency for the teaching of PC maintenance work was improved about three times that of the conventional method. 
- In the future, we plan to study a courseware creating method that supports the "Analysis of an object" (understanding of a PC manual).

\section{References}

[Fukuhara 91] Fukuhara, Y. (1991) Multimedia Authoring System for Practical Sceneoriented ITS (CAIRNEY): ICOMMET 91, pp. 215-218

[Fukuhara 93] Fukuhara, Y. (1993) CAI system (CAIRNEY) for tele-communication network operator: SIG-IES-9303-7, pp. 37-42

[Maruyama 95] Maruyama. (1995) Proposal of hyper link architecture in the distributed environment CALAT: AI symposium 95, JSAI

\section{Biography}

Y. Terahama is a Researcher of Systems Development Laboratory (S.D.L). He is engaged in the study of business performance and training support system.

H. Kojima is Senior Researcher of S.D.L. He is engaged in the study of business performance and training support. He holds a Ph.D. in Computer Application.

H. Yamamoto is a General Manager of Engineering Education Center. He is engaged in the study of CAI education support functions on self-study. 